\title{
ON $C R$-MAPPINGS BETWEEN ALGEBRAIC CAUCHY-RIEMANN MANIFOLDS AND SEPARATE ALGEBRAICITY FOR HOLOMORPHIC FUNCTIONS
}

\author{
RUSLAN SHARIPOV AND ALEXANDER SUKHOV
}

\begin{abstract}
We prove the algebraicity of smooth $C R$-mappings between algebraic Cauchy-Riemann manifolds. A generalization of separate algebraicity principle is established.
\end{abstract}

\section{INTRODUCTION}

One of the most surprising phenomena of geometric theory of several complex variables is the following one: every local biholomorphism of the (real) unit sphere of $\mathbb{C}^{n}, n>1$, extends to a global automorphism of the unit ball and, particularly, to a complex rational mapping of the whole space $\mathbb{C}^{n}$ (of course, this is not true in one variable). Poincaré $[\mathrm{Po}]$ was the first who obtained such result for $n=2$; later the general case was considered independently by Tanaka [Ta], Pelles $[\mathrm{Pe}]$ and Alexander $[\mathrm{Al}]$. We mention also a quite elementary and very elegant proof of the result of this type (in fact, more general) in Rudin's book [Ru].

Now there exist several generalizations of this classical result in different directions. Thus, Tumanov and Henkin [TH], Tumanov [Tu], Forstneric [Fo] and Sukhov $[\mathrm{Su} 1, \mathrm{Su} 3]$ investigated the rational extendability of (locally defined) $C R$-mappings between quadric Cauchy - Riemann manifolds of higher codimensions. On the other hand, for the case of hypersurfaces Webster in his famous paper [W1] discovered a very natural extension of the Poincaré phenomenon in the algebraic category: a local biholomorphism between two real algebraic Levi - nondegenerate hypersurfaces extends to an algebraic mapping on all $\mathbb{C}^{n}$. In the present paper we generalize this result to $C R$-mappings between real algebraic Cauchy - Riemann manifolds of higher codimensions (Theorem 1) (another generalization was obtained in [Su4]). Our technique is based on the Webster reflection principle modified in the spirit of [Su1] and a generalization of the classical separate algebraicity theorem, where we replace the parallel lines by families of algebraic curves (Theorem 2). We hope that this second result is of self-interest.

The paper is organized as follows. In section 2 we give the precise definitions and statements of our results. In sections 3 and 4 we prove Theorem 1 provided Theorem 2 holds. Section 5 is devoted to the proof of Theorem 2.

Received by the editors January 26, 1995.

1991 Mathematics Subject Classification. Primary 32D15, 32D99, 32F25, 32H99.

Key words and phrases. $C R$-mapping, reflection principle, algebraic $C R$-manifold, separate algebraicity.

Paper written under the financial support of the International Scientific Foundation of Soros, project \#RK4000 


\section{The Results}

Let $\Omega$ be a domain in $\mathbb{C}^{n}$. A closed subset $M$ of $\Omega$ is called a generic real algebraic manifold of codimension $d \geq 1$ if

$$
M=\left\{z \in \Omega: \rho_{j}(z, \bar{z})=0, j=1, \ldots, d\right\},
$$

where $\rho_{j}$ are real polynomials and $\bar{\partial} \rho_{1} \wedge \ldots \wedge \bar{\partial} \rho_{d} \neq 0$ in $\Omega$. They are called the defining functions of $M$. We denote by $T_{p} M$ and $T_{p}^{c} M$ the real and complex tangent spaces of $M$ at a point $p \in M$ (recall that $T_{p}^{c} M=T_{p} M \cap J\left(T_{p} M\right)$, where $J$ is the complex structure operator in $\left.\mathbb{C}^{n}\right)$. This is well known that for a generic manifold the complex dimension of $T_{p}^{c} M$ does not depend on $p \in M$ and is equal to $n-d$; it is called the $C R$-dimension of $M$.

We denote by $H_{p}\left(\rho_{j}, u, v\right)$ the value of the Levi form (complex hessian) of the function $\rho_{j}$ on vectors $u, v$ at the point $p \in M$, i.e.

$$
H_{p}\left(\rho_{j}, u, v\right)=\sum_{\nu, \mu=1}^{n} \frac{\partial^{2} \rho_{j}}{\partial z_{\nu} \partial \bar{z}_{\mu}}(p) u_{\nu} \bar{v}_{\mu} .
$$

The Levi cone (at $p \in M$ ) of the manifold $M$ of the form (2.1) is said to be the convex hull of the set

$$
\left\{\alpha=\left(\alpha_{1}, \ldots, \alpha_{d}\right) \in \mathbb{R}^{d}: \alpha_{j}=H_{p}\left(\rho_{j}, u, u\right), u \in T_{p}^{c} M\right\} .
$$

If the Levi cone of $M$ has a non-empty interior in $\mathbb{R}^{d}$, then we say that $M$ possess a non-degenerate Levi cone at $p$. Evidently this condition does not depend on the choice of defining functions and is invariant with respect to changes of coordinates.

The vector valued hermitian form

$$
L_{p}(u, v)=\left(H_{p}\left(\rho_{1}, u, v\right), \ldots, H_{p}\left(\rho_{d}, u, v\right)\right)
$$

is called the Levi form of $M$ at $p$.

Along with $M$ we consider a domain $\Omega^{\prime}$ in $\mathbb{C}^{n^{\prime}}$ and a generic real algebraic manifold $M^{\prime}$ of codimension $d^{\prime} \geq 1$ of the form

$$
M^{\prime}=\left\{z^{\prime} \in \Omega^{\prime}: \rho_{j}^{\prime}\left(z^{\prime}, \bar{z}^{\prime}\right)=0, j=1, \ldots, d^{\prime}\right\},
$$

where $\rho_{j}^{\prime}$ are real polynomials and $\bar{\partial} \rho_{1}^{\prime} \wedge \ldots \wedge \bar{\partial} \rho_{d^{\prime}}^{\prime} \neq 0$ in $\Omega^{\prime}$. Fix a hermitian scalar product in $\mathbb{C}^{n^{\prime}}$ that is denoted by $\langle\cdot, \cdot\rangle^{\prime}$. Fix a point $p^{\prime} \in M^{\prime}$. With every defining function $\rho_{j}^{\prime}$ we associate the Levi operator $L_{p^{\prime}}^{j}: T_{p^{\prime}}^{c} M^{\prime} \longrightarrow T_{p^{\prime}}^{c} M^{\prime}$ determined by $H_{p^{\prime}}\left(\rho_{j}^{\prime}, u, v\right)=\left\langle L_{p^{\prime}}^{j}(u), v\right\rangle^{\prime}$ for all $u, v \in T_{p^{\prime}}^{c} M^{\prime}$. Of course, this definition of the Levi operator depends on the choice of hermitian scalar product. But as we shall see further this dependence is inessential. In what follows we use the similar notation for the Levi operators of $M$ (without primes).

Let $U$ be an open connected subset of the manifold $M$. The mapping $F: U \longrightarrow$ $M^{\prime}$ of the smoothness class $C^{1}$ is called a $C R$-mapping if for any point $p \in U$ the tangent map $d F_{p}$ is $\mathbb{C}$-linear after restriction to the complex tangent space $T_{p}^{c} M$ (in this case $\left.d F_{p}\left(T_{p}^{c} M\right) \subset T_{p^{\prime}}^{c} M^{\prime}\right)$. We say that $F$ extends to an algebraic mapping of all $\mathbb{C}^{n}$ if the graph of $F$ is a part of $n$-dimensional complex algebraic manifold in $\mathbb{C}^{n+n^{\prime}}$.

Now we can formulate the first main result of our paper. It is given by the following theorem. 
Theorem 1. Let $\Omega \in \mathbb{C}^{n}$ and $\Omega^{\prime} \in \mathbb{C}^{n^{\prime}}$ be domains, $M \subset \Omega$ and $M^{\prime} \subset \Omega^{\prime}$ be generic real algebraic manifolds of the form (2.1) and (2.4) respectively, $M$ having the non-degenerate Levi cone at some point $p \in M$. Suppose $U \subset M$ is an open connected subset of $M$ containing $p$ and let $F: U \longrightarrow M^{\prime}$ be a CR-mapping of class $C^{1}$ satisfying the following condition

$$
\sum_{j=1}^{d^{\prime}} L_{p^{\prime}}^{j}\left(d F_{p}\left(T_{p}^{c} M\right)\right)=T_{p^{\prime}}^{c} M^{\prime}, \text { where } p^{\prime}=F(p) .
$$

Then $F$ extends to an algebraic mapping of the whole space $\mathbb{C}^{n}$.

We note first that (2.5) does not depend on the choice of the hermitian scalar product in $\mathbb{C}^{n^{\prime}}$ by means of which we defined the Levi operators $L_{p^{\prime}}^{j}$. If $\tilde{L}_{p^{\prime}}^{j}$ is defined by another scalar product, then it is connected with $L_{p^{\prime}}^{j}$ by the equality $\tilde{L}_{p^{\prime}}^{j}=A L_{p^{\prime}}^{j}$, where $A$ is a non-degenerate $\mathbb{C}$-linear operator in $T_{p^{\prime}}^{c} M^{\prime}$. Therefore (2.5) holds for operators $\tilde{L}_{p^{\prime}}^{j}$ as well.

Recall that the Levi form of $M$ is called non-degenerate if $L_{p}(u, v)=0$ for any $v \in T_{p}^{c} M$ implies $u=0$ [W2].

Corollary. Let $F: M \longrightarrow M^{\prime}$ be a $C R$ diffeomorphism of class $C^{1}$ between two real algebraic manifolds in $\mathbb{C}^{n}$ with non-degenerate Levi forms and non-degenerate Levi cones. Then $F$ extends to an algebraic mapping on all $\mathbb{C}^{n}$.

This assertion follows from Theorem 1 quite similarly to [Su1]. We emphasize that Theorem 1 treats a considerably more general situation, since $M$ and $M^{\prime}$ are allowed to have different $C R$ dimensions. Our proof of Theorem 1 is based on the modification of the Webster reflection principle [W1]. The crucial technical tool here is a "curved" version of the following classical separate algebraicity principle.

Claim. Let $f(\mathbf{z})=f\left(z_{1}, \ldots, z_{n}\right)$ be a function in some domain $D \subset \mathbf{C}^{n}$. If $f(\mathbf{z})$ is algebraic in each separate variable $z_{i}$ for any fixed values of other variables, then $f(\mathbf{z})$ is an algebraic function in $D$.

Proof of this classical theorem can be found in $[\mathrm{BM}]$. Function $f(\mathbf{z})$ in this assertion is algebraic along the straight lines parallel to the coordinate axes. The domain $D$ foliates into $n$ families of such lines. In order to generalize this result for our purposes we introduce $n$ families of algebraic curves in $D$

$$
\begin{aligned}
& z_{1}=R_{1}^{(m)}\left(t_{m}, c_{1}^{(m)}, \ldots, c_{n-1}^{(m)}\right), \\
& \text {. . . . . . . . . . . . } \\
& z_{n}=R_{n}^{(m)}\left(t_{m}, c_{1}^{(m)}, \ldots, c_{n-1}^{(m)}\right) .
\end{aligned}
$$

Here $m=1, \ldots, n$ is the number of the family, $t_{m}$ is a parameter on each particular curve of the $m$-th family $\left(R_{i}^{(m)}\right.$ depends on $t_{m}$ algebraically). Parameters $c_{1}^{(m)}, \ldots, c_{n-1}^{(m)}$ identify curves of the $m$-th family.

Definition 1. The family of algebraic curves (2.6) is called algebraically depending on parameters if each of the defining functions $R_{i}^{(m)}, i=1, \ldots, n$, in $(2.6)$ is algebraic in $c_{1}^{(m)}, \ldots, c_{n-1}^{(m)}$. 
In this case because of the above-mentioned classical separate algebraicity principle functions $R_{i}^{(m)}$ in (2.6) are algebraic in the whole set of their arguments $t_{m}, c_{1}^{(m)}, \ldots, c_{n-1}^{(m)}$.

Definition 2. The family of curves (2.6) is called nonsingular in $D$ if the curves of this family fill the whole domain $D$ and the mapping $R^{(m)}:\left(t_{m}, c_{1}^{(m)}, \ldots, c_{n-1}^{(m)}\right) \longrightarrow$ $\mathbf{z}$ is a local diffeomorphism.

Definition 3. Let (2.6) define $n$ nonsingular families of algebraic curves in $D$. Then we have $n$ tangent vectors to the curves at each point of $D$. We shall say that $n$ families of curves (2.6) are in general position if these vectors are linearly independent at any point $\mathbf{z} \in D$.

Now we can state the "curved" separate algebraicity principle which partially generalizes the classical one and forms the second main result of our paper.

Theorem 2. Let $D \subset \mathbb{C}^{n}$ be a domain equipped with $n$ families (2.6) of nonsingular algebraic curves algebraically depending on parameters and being in general position. Then each holomorphic function $f(\mathbf{z})$ in $D$, which is algebraic in $t_{m}$ after restriction to any particular curve from any one of these families, extends to an algebraic function on $\mathbb{C}^{n}$.

\section{TANGent $C R$-FIELDS AND THE MAIN EQUATIONS}

First we shall recall briefly some facts of the theory of $C R$-structures (reader can find more details in $[\mathrm{Ch}])$. Let $\left(T_{p}^{c} M\right)_{\mathbb{R}}$ be the complex tangent space $T_{p}^{c} M$ considered as a vector space over real numbers $\mathbb{R}$. Then $\left(T_{p}^{c} M\right)_{\mathbb{R}} \stackrel{\mathbb{R}}{\otimes} \mathbb{C}$ is a complexification for $\left(T_{p}^{c} M\right)_{\mathbb{R}}$. The operator $J$ of canonical complex structure in this complexification is $\mathbb{C}$-linear with respect to the own complex structure of $T_{p}^{c} M$. Therefore it has two eigenvalues $+i$ and $-i$. Then we have the decomposition $\left(T_{p}^{c} M\right)_{\mathbb{R}} \otimes \mathbb{C}=T_{p}^{c} M^{1,0} \oplus T_{p}^{c} M^{0,1}$, where

$$
T_{p}^{c} M^{1,0}=\left\{(v,-i v): v \in T_{p}^{c} M\right\} \text { and } T_{p}^{c} M^{0,1}=\left\{(v,+i v): v \in T_{p}^{c} M\right\}
$$

are the eigenspaces for $J$ corresponding to the eigenvalues $+i$ and $-i$ respectively. The following two maps $v \longmapsto(v,-i v)$ and $v \longmapsto(v,+i v)$ realize the canonical isomorphisms $T_{p}^{c} M \equiv T_{p}^{c} M^{1,0}$ and $\overline{T_{p}^{c} M} \equiv T_{p}^{c} M^{0,1}$. Thus we have $T_{p}^{c} M_{\mathbb{R}} \otimes$ $\mathbb{C}=T_{p}^{c} M \oplus \overline{T_{p}^{c} M}$. Because of the last decomposition the sections of the vector bundles $T^{c} M$ and $\overline{T^{c} M}$ are called the $C R$ vector fields of the types $(1,0)$ and $(0,1)$ respectively. It is easy to check that the field $V=\sum_{j=1}^{n} v_{j}(z) \partial / \partial z_{j}$ in $\mathbb{C}^{n}$ is the vector field of the type $(1,0)$ on $M$ if and only if the vector $\left(v_{1}(a), \ldots, v_{n}(a)\right)$ is in $T_{a}^{c} M$ for any $a \in M$.

Let us go back to Theorem 1. Note that without loss of generality we can take $p=0$ and $F(p)=0$. Taking $z=(x, y)$ and $z^{\prime}=\left(x^{\prime}, y^{\prime}\right)$ and denoting $\mathbb{C}^{n}=\mathbb{C}_{x}^{k} \times \mathbb{C}_{y}^{d}$ and $\mathbb{C}^{n^{\prime}}=\mathbb{C}_{x^{\prime}}^{k^{\prime}} \times \mathbb{C}_{y^{\prime}}^{d^{\prime}}$ one can bring the defining polynomials for $M$ and $M^{\prime}$ to the form

$$
\begin{aligned}
& p_{j}=y_{j}+\bar{y}_{j}+o(|z|), \quad j=1, \ldots, d, \\
& p_{j}^{\prime}=y_{j}^{\prime}+\bar{y}_{j}^{\prime}+o(|z|), \quad j=1, \ldots, d^{\prime} .
\end{aligned}
$$


ON CR-MAPPINGS BETWEEN ALGEBRAIC CAUCHY-RIEMANN MANIFOLDS 771

For this choice of coordinates we have $T_{0}^{c} M=\mathbb{C}_{x}^{k}=\{(x, y): y=0\}$ and $T_{0}^{c} M^{\prime}=$ $\mathbb{C}_{x^{\prime}}^{k^{\prime}}=\left\{\left(x^{\prime}, y^{\prime}\right): y^{\prime}=0\right\}$. Now let us consider vector fields $T_{q}, q=1, \ldots, k$, of the form

$$
T_{q}=\Delta(z, \bar{z}) \frac{\partial}{\partial x_{q}}-\sum_{j=1}^{d} a_{j q}(z, \bar{z}) \frac{\partial}{\partial y_{j}}
$$

where $\Delta$ is the determinant of the matrix

$$
\Delta_{s j}=\left(\frac{\partial \rho_{s}}{\partial y_{j}}\right)_{s=1, \ldots, d}^{j=1, \ldots, d}
$$

Everywhere in this paper we shall obey the following rule for denoting the matrix elements: lower index outside the right bracket is the row number and upper index is the column number. The coefficients $a_{j q}$ in (3.4) we define as follows

$$
a_{j q}=\sum_{s=1}^{d} \Delta b_{j s} \frac{\partial \rho_{s}}{\partial x_{q}}
$$

where $b_{j s}$ is the inverse of the matrix (3.3). According to elementary facts from linear algebra the matrix with the elements $\Delta b_{j s}$ is a conjugate matrix for (3.3), i.e. its elements are the algebraic cofactors for the elements of the transpose of (3.3). Therefore the coefficients of the vector fields in (3.2) are polynomials in $x_{i}$ and $y_{i}$.

Clearly, the restrictions of the fields $T_{q}, q=1, \ldots, k$, form a base of the bundle $T^{c} M$ over a neighborhood of the origin in $M$. Moreover, it is obvious that

$$
\Delta(0)=1, \quad a_{j q}(0)=0, \quad j=1, \ldots, d, q=1, \ldots, k .
$$

Note that because of $d F_{0}\left(T_{0}^{c} M\right) \subset T_{0}^{c} M^{\prime}$, we get by (3.1)

$$
\partial F_{j} / \partial x_{q}(0)=0, \quad j=k^{\prime}+1, \ldots, n^{\prime}, q=1, \ldots, k .
$$

Together with the fields (3.2) let us consider the conjugate fields $\overline{T_{q}}$. Recall that a $C^{1}$-function $h$ defined on an open connected subset $U \subset M$ is called a $C R$-function if for any $z=(x, y) \in U$ one has

$$
\overline{T_{q}} h=\Delta \frac{\partial h}{\partial \bar{x}_{q}}-\sum_{j=1}^{d} \bar{a}_{j q} \frac{\partial h}{\partial \bar{y}_{j}}=0, \quad q=1, \ldots, k .
$$

These are the tangent Cauchy-Riemann equations. It is well known that a $C^{1}$ mapping $F: M \longrightarrow M^{\prime}$ is a $C R$-mapping (in the sense of section 2) if and only if each its component is a $C R$-function on $M$. Indeed, (3.7) means that $\bar{\partial} h\left(V^{q}\right)=$ $0, q=1, \ldots, k$, (where $\left\{V^{q}\right\}_{q=1}^{k}$ is a base of $\left.T_{p}^{c} M\right)$ for any point $p \in M$. Therefore $\bar{\partial} h \mid T_{p}^{c} M=0$ and (3.7) means that the restriction $d h \mid T_{p}^{c} M$ is a $\mathbb{C}$-linear function for any $p \in M$. 
According to the Boggess-Polking theorem $[\mathrm{BP}]$ from the non-degeneracy of the Levi cone of $M$ at the origin $0 \in M$ we derive that the mapping $F$ extends holomorphically to a wedge with the edge $M$. Using the results of [Su1, Su2] we obtain that $F$ extends holomorphically to a neighborhood of the origin in $\mathbb{C}^{n}$. Therefore everywhere below we take $F$ being holomorphic in a neighborhood $\Omega \ni 0$ in $\mathbb{C}^{n}$ and suppose $U=M \cap \Omega$.

The condition $F(U) \subset M^{\prime}$ means that $\rho_{j}^{\prime}(F, \bar{F})=0$ for $z \in U$ and $j=1, \ldots, d^{\prime}$. Applying the tangent operators (3.2) to both sides of these equalities we obtain

$$
T_{q} \rho_{j}^{\prime}(F, \bar{F})=0, \quad q=1, \ldots, k, j=1, \ldots, d^{\prime} \text { for } z \in U .
$$

Now we introduce the vector-function $D(F)$ holomorphic in $\Omega$

$$
D(F)=\left(\frac{\partial F_{1}}{\partial z_{1}}, \ldots, \frac{\partial F_{n^{\prime}}}{\partial z_{1}}, \ldots, \frac{\partial F_{1}}{\partial z_{n}}, \ldots, \frac{\partial F_{n^{\prime}}}{\partial z_{n}}\right)
$$

Its values are in $\mathbb{C}^{n n^{\prime}}$.

The left-hand sides of (3.8) can be considered as polynomials in $z, \bar{z}, F, \bar{F}$ and $\partial F_{j} / \partial z_{s}, \partial \bar{F}_{j} / \partial z_{s}$. But since $F$ is holomorphic, we have $\partial \bar{F} / \partial z_{s}=0$. Thus, the following expressions

$$
\Phi_{q j}(z, \bar{z}, F, \bar{F}, D(F))=T_{q} \rho_{j}^{\prime}(F, \bar{F})
$$

are polynomials in $z, \bar{z}, F, \bar{F}, D(F)$. By $\tilde{0}$ we denote the point $(0,0,0,0, D(F)(0))$ in $\mathbb{C}^{n} \times \mathbb{C}^{n} \times \mathbb{C}^{n^{\prime}} \times \mathbb{C}^{n^{\prime}} \times \mathbb{C}^{n n^{\prime}}$.

Lemma 3.1. In the set of functions (3.9) with $q=1, \ldots, k, j=1, \ldots, d^{\prime}$ one can choose a subset $\Phi_{1}=\Phi_{q(1) j(1)}, \ldots, \Phi_{k^{\prime}}=\Phi_{q\left(k^{\prime}\right) j\left(k^{\prime}\right)}$ such that the following Jacobian matrix is of rank $k^{\prime}$

$$
\left(\frac{\partial \Phi_{j}}{\partial \bar{F}_{s}}(\tilde{0})\right)_{j=1, \ldots, k^{\prime}}^{s=1, \ldots, k^{\prime}}
$$

Proof. Let us fix $j$, consider the set of functions $\Phi_{q j}, q=1, \ldots, k$, and form the Jacobian matrix

$$
\left(\frac{\partial \Phi_{q j}}{\partial \bar{F}_{s}}(\tilde{0})\right)_{j=1, \ldots, k^{\prime}}^{s=1, \ldots, k} .
$$

It follows from (3.1), (3.2), (3.5), (3.6), (3.8), (3.9) that this matrix can be rewritten in the form

$$
\left(\sum_{r=1}^{k^{\prime}} \frac{\partial^{2} \rho_{j}^{\prime}}{\partial z_{r}^{\prime} \partial \bar{z}_{s}^{\prime}}(0) \frac{\partial F_{r}}{\partial z_{q}}(0)\right)_{j=1, \ldots, k^{\prime}}^{s=1, \ldots, k}
$$

The condition (2.5) does not depend on the choice of scalar product in $\mathbb{C}^{n^{\prime}}$. Therefore one can take the hermitian scalar product, which defines Levi operators 
ON $C R$-MAPPINGS BETWEEN ALGEBRAIC CAUCHY-RIEMANN MANIFOLDS 773

$L_{0}^{j}$, being canonical in the coordinates (3.1). Then each operator $L_{0}^{j}$ in the standard basis $e_{r}^{\prime}, r=1, \ldots, k^{\prime}$, of $\mathbb{C}^{k^{\prime}}=T_{0}^{c} M^{\prime}$ has the matrix of the following form

$$
\left(\frac{\partial^{2} \rho_{j}^{\prime}}{\partial z_{j}^{\prime} \partial \bar{z}_{s}^{\prime}}(0)\right)_{j=1, \ldots, k^{\prime}}^{s=1, \ldots, k^{\prime}} .
$$

Therefore the $q$-th row of the matrix (3.11) consists of the coordinates of the vector $L_{0}^{j}\left(d F_{0}\left(e_{q}\right)\right)$, where $e_{q}, q=1, \ldots, k$, is the standard basis of $\mathbb{C}^{k^{\prime}}=T_{0}^{c} M^{\prime}$, i.e.

$$
{ }^{t}\left(\partial \Phi_{q j} / \partial \bar{F}_{1}(\tilde{0}), \ldots, \partial \Phi_{q j} / \partial \bar{F}_{k}(\tilde{0})\right)=L_{0}^{j}\left(d F_{0}\left(e_{q}\right)\right)
$$

where ${ }^{t}()$ denotes the transpose. According to (2.5) the rank of the set of vectors $L_{0}^{j}\left(d F_{0}\left(e_{q}\right)\right), j=1, \ldots, d^{\prime}, q=1, \ldots, k$, is equal to $k$. Thus we get the proof of the lemma.

Let $\Phi_{j}, j=1, \ldots, k^{\prime}$, be the functions chosen according to Lemma 3.1. For $z \in U$ we have

$$
\begin{aligned}
& \Phi_{j}(z, \bar{z}, F, \bar{F}, D(F))=0, \quad j=1, \ldots, k^{\prime}, \\
& \rho_{s}^{\prime}(F, \bar{F})=0, \quad s=1, \ldots, d^{\prime} .
\end{aligned}
$$

Lemma 3.2. The rank of the Jacobian matrix for (3.12) with respect to $\bar{F}$ at the point $\tilde{0}$ is equal to $n^{\prime}$.

Proof. The Jacobian matrix mentioned in the lemma has the form

$$
\left(\begin{array}{c|c}
\frac{\partial \Phi_{j}(\tilde{0})}{\partial \bar{F}_{s}} & * \\
\hline \tilde{0}_{d^{\prime}}^{k^{\prime}} & I_{d^{\prime}}
\end{array}\right) .
$$

The upper left block of the matrix (3.13) is formed by the matrix (3.10), $\mathbf{0}$ is the zero $d^{\prime} \times k^{\prime}$ matrix and $I$ is a unit $d^{\prime} \times d^{\prime}$ matrix. Therefore by Lemma 3.1 the rank of (3.13) is equal to $k^{\prime}+d^{\prime}=n^{\prime}$.

Applying the complex conjugation to the first $k^{\prime}$ equations (3.13), we get

$$
\begin{aligned}
& P_{j}(z, \bar{z}, F, \bar{F}, \overline{D(F)})=0, \quad j=1, \ldots, k^{\prime}, \\
& \rho_{s}^{\prime}(F, \bar{F})=0, \quad s=1, \ldots, d^{\prime},
\end{aligned}
$$

where $P_{j}$ are polinomials in $z, \bar{z}, F, \bar{F}, \bar{D}(F)$. This system of equations is of crucial importance in what follows.

\section{Geometry of Segre surfaces}

For a real algebraic manifold $M$ of the form (2.1) the Segre surface associated to a fixed point $z \in \mathbb{C}^{n}$ is a complex algebraic set in $\mathbb{C}^{n}$ of the form

$$
Q(z)=\left\{w \in \mathbb{C}^{n}: \rho_{j}(w, \bar{z})=0, j=1, \ldots, d\right\} .
$$

We denote by $A$ the graph of the mapping $F$ over a neighborhood $\Omega \ni 0$ in $\mathbb{C}^{n}$. Also let

$$
A_{\zeta}=\left\{\left(z, z^{\prime}\right) \in \mathbb{C}^{n+n^{\prime}}: z^{\prime}=F(z), \rho_{j}(z, \bar{\zeta})=0, j=1, \ldots, d\right\}
$$

denote the graph of the restriction of $F$ to the Segre surface $Q(\zeta)$. Evidently, every $A_{\zeta}$ is a $k(=n-d)$-dimensional complex manifold in $\Omega \times \mathbb{C}^{n^{\prime}}$. 
Lemma 4.1. For any point $\zeta \in \Omega$ the complex manifold $A_{\zeta}$ is a piece of a complex $k$-dimensional algebraic variety $\tilde{A}_{\zeta}$ in $\mathbb{C}^{n+n^{\prime}}$.

Proof. It follows from Lemma 3.2 that one can apply the implicit function theorem to the system (3.14). We get $F(z)=R(z, \bar{z})$ for $z \in M \cap \Omega$ (where $R$ is a real analytic function in $\Omega$ algebraic in $z$ ). By (3.1) and the implicit function theorem we get $M \cap \Omega=\{z=(x, y) \in \Omega: y=\phi(x, \bar{z})\}$. Therefore,

$$
F(x, \phi(x, \bar{z}))=R(x, \phi(x, \bar{z}), \bar{z})
$$

for $z=(x, y) \in M \cap \Omega$. Consider antiholomorphic functions $F^{\star}(\theta, \xi)=F(\bar{\theta}, \phi(\bar{\theta}, \bar{\xi}))$ and $R^{\star}(\theta, \xi)=R(\bar{\theta}, \phi(\bar{\theta}, \bar{\xi}), \bar{\xi})$, where $\theta \in \mathbb{C}^{k}, \xi \in \mathbb{C}^{n}$. Then (4.3) means that these functions coincide on the manifold

$$
\hat{M}=\left\{(\theta, \xi): \bar{\theta}=\left(\xi_{1}, \ldots, \xi_{k}\right), \xi \in M\right\}
$$

which is obviously generic in a neighborhood of the origin in $\mathbb{C}^{n+k}$. Now it follows from the uniqueness theorem $[\mathrm{Pi}]$ that $F(\bar{\theta}, \phi(\bar{\theta}, \bar{\xi})) \equiv R(\bar{\theta}, \phi(\bar{\theta}, \bar{\xi}), \bar{\xi})$ in a neighborhood of the origin in $\mathbb{C}^{n+k}$. Hence,

$$
F(x, \phi(x, \bar{\zeta}))=R(x, \phi(x, \bar{\zeta}), \bar{\zeta})
$$

for any fixed $\zeta$ in a neighborhood of the origin in $\mathbb{C}^{n}$. But the set

$$
\{(x, \phi(x, \bar{\zeta})): x \text { runs over a neighborhood of the origin }\}
$$

coincides with the Segre surface $Q(\zeta)=\left\{z: \rho_{j}(z, \bar{\zeta})=0, j=1, \ldots, d\right\}$. Thus we get $F(z)=R(z, \bar{\zeta})$ for $z \in Q(\zeta)$. Since $R$ was obtained by (3.14), the set

$$
A_{\zeta}=\left\{\left(z, z^{\prime}\right): z \in Q(\zeta), z^{\prime}=F(z)\right\}
$$

is contained in an $(n-d)$-dimensional complex algebraic variety of the form

$$
\begin{aligned}
& P_{j}\left(z, \bar{\zeta}, z^{\prime}, \bar{F}(\zeta), \overline{D F(\zeta)}\right)=0, \quad j=1, \ldots, k^{\prime}, \\
& \rho_{s}^{\prime}\left(z^{\prime}, \bar{F}(\zeta)\right)=0, \quad s=1, \ldots, d^{\prime}, \\
& \rho_{l}(z, \bar{\zeta})=0, \quad l=1, \ldots, d,
\end{aligned}
$$

proves the desired assertion. $\mathbb{C}^{d}$.

Fix $\theta \in \mathbb{C}^{k}$ and consider the $d$-parametric family of Segre surfaces $Q(\theta, \tau), \tau \in$

Lemma 4.2. There exists a neighborhood $U \ni 0$ in $\mathbb{C}^{n}$ of the form $U=U_{x} \times U_{y}$, $U_{x} \subset \mathbb{C}^{k}, U_{y} \subset \mathbb{C}^{d}$ such that for any fixed $\theta \in U_{x}$, the family of Segre surfaces $Q(\theta, \tau), \tau \in U_{y}$, has the following properties:

(1) for any $\tau^{\prime}, \tau^{\prime \prime} \in U_{y}$ the intersection $Q\left(\theta, \tau^{\prime}\right) \cap Q\left(\theta, \tau^{\prime \prime}\right)$ is empty;

(2) for any $z=(x, y) \in U$ there exists a unique $\tau \in U_{y}$ such that $(x, y) \in Q(\theta, \tau)$. 
ON $C R$-MAPPINGS BETWEEN ALGEBRAIC CAUCHY-RIEMANN MANIFOLDS 775

Proof. One can represent the Segre surface as $Q(\theta, \tau)=\{z \in U: \bar{\tau}=S(z, \bar{\theta})\}$, where $S$ is an analytic function and $U$ is a neighborhood of the origin. Now, if $z=(x, y)$ is in $Q\left(\theta, \tau^{\prime}\right) \cap Q\left(\theta, \tau^{\prime \prime}\right)$, then $\bar{\tau}^{\prime}=S(z, \bar{\theta})=\bar{\tau}^{\prime \prime}$; this implies (1). For $z=(x, y)$ we set $\tau=\bar{S}(z, \bar{\theta})$. Then $z \in Q(\theta, \tau)$ and we get (2).

By the implicit function theorem

$$
Q(\theta, \tau) \cap U=\{(x, y) \in U: y=R(x, \bar{\theta}, \bar{\tau})\},
$$

where $R$ is an algebraic function, i.e. locally $Q(\theta, \tau)$ is the graph over the coordinate plane $\mathbb{C}_{x}^{k}=\mathbb{C}_{z_{1} \ldots z_{k}}^{k}$. Let $X_{(\theta, \tau)}^{j}$ be holomorphic vector fields on $Q(\theta, \tau)$, the natural liftings to $Q(\theta, \tau)$ of the coordinate vector fields $\partial / \partial z_{j}, j=1, \ldots, k$ in $\mathbb{C}_{z_{1} \ldots z_{k}}^{k}$. It follows from Lemma 4.2 that for any point $(x, y) \in U$ there exists the unique surface $Q(\theta, \tau), \tau=\bar{S}(z, \bar{\theta})$ passing through $(x, y)$. Hence, one can consider the holomorphic vector fields $Y_{(\theta)}^{j}(z)$ (depending on the parameter $\theta$ ) in $U$ defined as follows: given $z$ in $U$ and fixed $\theta$ we set $\tau=\bar{S}(z, \bar{\theta})$ and $Y_{(\theta)}^{j}(z)=X_{(\theta, \tau)}^{j}(z)$. Their integral curves evidently are linear sections of the Segre surfaces by parallel planes and, therefore, form families of complex algebraic curves in $\mathbb{C}^{n}$ algebraically depending on parameters.

Lemma 4.3. The set of vectors $Y_{(\theta)}^{j}(0), j=1, \ldots, k, \theta$ runs over a neighborhood of the origin in $\mathbb{C}^{k}$, spans $\mathbb{C}^{n}$.

Proof. If $(\theta, \tau) \in Q(0)$, i.e. $\rho_{j}(\theta, \tau, 0,0)=0, j=1, \ldots, d$, then it follows from (3.1) and the implicit function theorem that $\tau=o(|\theta|)$. Now let $0 \in Q(\theta, \tau)$ (recall that this is equivalent to $(\theta, \tau) \in Q(0))$. By the implicit function theorem we get

$$
Q(\theta, \tau)=\{(x, y): y+\bar{\tau}=\phi(x, \bar{\theta}, y, \bar{\tau})\}=\{(x, y): y+\bar{\tau}=\psi(x, \bar{\theta}, \bar{\tau})\} .
$$

Since $\tau=o(|\theta|)$, we have

$$
\psi=\langle L(x), \theta+o(|\theta|)\rangle+o(|\theta|)+o(|x|),
$$

where

$$
\langle L(\xi), \eta\rangle=\left(\left\langle L_{1}(\xi), \eta\right\rangle, \ldots,\left\langle L_{d}(\xi), \eta\right\rangle\right)
$$

is the Levi form of $M, L_{j}$ being the Levi operators of $M$ at the origin. Hence

$$
Y_{(\theta)}^{j}(0)=\left(e_{j} \mid\left\langle L\left(e_{j}\right), \theta\right\rangle+o(|\theta|)\right)=\left(0, \ldots, 1, \ldots, 0,\left\langle L\left(e_{j}\right), \theta\right\rangle+o(|\theta|)\right),
$$

where 1 is on the $j$-th position and $e_{j}, j=1, \ldots, k$, is the standard basis of $\mathbb{C}^{k}$.

Assume there exists $\alpha \in \mathbb{C}^{n} \backslash\{0\}$ such that

$$
\left\langle\alpha, Y_{(\theta)}^{j}(0)\right\rangle=0, \quad j=1, \ldots, k,
$$

for any $\theta \in U_{x}$. Then

$$
\begin{aligned}
\left\langle\alpha, Y_{(\theta)}^{j}(0)\right\rangle & =\alpha_{j}+\sum_{\nu=1}^{d}\left(\left\langle L_{\nu}\left(e_{j}\right), \theta\right\rangle+o(|\theta|)\right) \alpha_{k+\nu} \\
& =\alpha_{j}+\sum_{\nu=1}^{d} \alpha_{k+\nu}\left\langle L_{\nu}\left(e_{j}\right), \theta\right\rangle+o(|\theta|) \\
& =\alpha_{j}+\left\langle\sum_{\nu=1}^{d} \alpha_{k+\nu} L_{\nu}\left(e_{j}\right), \theta\right\rangle+o(|\theta|) \equiv 0
\end{aligned}
$$


as a function of $\theta \in \mathbb{C}^{k}$. Therefore $\alpha_{j}=0$ and $\sum_{\nu=1}^{d} \alpha_{k+\nu} L_{\nu}\left(e_{j}\right)=0$ for $j=$ $1, \ldots, k$. This means that the Levi operators of $M$ are linearly dependent. We get a contradiction with the condition of non-degeneracy of the Levi cone of $M$.

Thus, we get $n$ non-singular families of algebraic curves, algebraically depending on the parameters, in general position near the origin and the restriction of $F$ to each curve is algebraic. Now it follows from Theorem 2 that $F$ extends to an algebraic mapping on all $\mathbb{C}^{n}$. This completes the proof of Theorem 1 provided Theorem 2 holds.

\section{Proof of Theorem 2}

First consider only the $m$-th family of algebraic curves (2.6). Because of nonsingularity of this family we can treat $t_{m}, c_{1}^{(m)}, \ldots, c_{n-1}^{(m)}$ in $(2.6)$ as new local coordinates in the domain $D$. Let us define the transformation $\varphi_{m}(\tau)$ as a translation along the $t_{m}$-axis in the new curvilinear coordinates

$$
\varphi^{(m)}(\tau): t_{m}, c_{1}^{(m)}, \ldots, c_{n-1}^{(m)} \longrightarrow t_{m}+\tau, c_{1}^{(m)}, \ldots, c_{n-1}^{(m)}
$$

The transformations $\varphi^{(m)}(\tau)$ form a local one-parameter group of transformations determined by the vector field of tangent vectors to the curves of the $m$-th family. In the original variables transformations (5.1) are given by $n$ algebraic functions of $n+1$ arguments

$$
\begin{gathered}
\tilde{z}_{1}=\varphi_{1}^{(m)}\left(\tau, z_{1}, \ldots, z_{n}\right), \\
\cdot . \cdot \cdot \cdot \cdot \cdot \cdot \cdot \\
\tilde{z}_{n}=\varphi_{n}^{(m)}\left(\tau, z_{1}, \ldots, z_{n}\right) .
\end{gathered}
$$

The algebraicity of the functions $\varphi_{i}^{(m)}$ in $(5.2)$ is a consequence of algebraicity of the curves of the $m$-th family and of their algebraic dependence on the parameters in $(2.6)$.

Using the transformations $z \longmapsto \tilde{z}=\varphi^{(m)}(\tau) z$ of the form (5.2) we introduce new local holomorphic coordinates $t_{1}, \ldots, t_{n}$ in $D$ as follows

$$
z=\varphi^{(n)}\left(t_{n}\right) \circ \ldots \circ \varphi^{(1)}\left(t_{1}\right) z^{0}
$$

where $z^{0}$ is a fixed point in whose neighborhood these coordinates are defined (recall that our families of curves are in general position). The transformation from $z_{1}, \ldots, z_{n}$ to $t_{1}, \ldots, t_{n}$ and the inverse are algebraic.

Note that part of coordinate lines in the local coordinates $t_{1}, \ldots, t_{n}$ coincide with the curves of the above families. Let $f\left(t_{1}, \ldots, t_{n}\right)$ be the representation of the function $f(z)$ from Theorem 2 in the local coordinates $t_{1}, \ldots, t_{n}$. Then the functions

$$
\begin{aligned}
f_{1} & =f(t, 0, \ldots, 0), \\
f_{2} & =f\left(t_{1}, t, 0, \ldots, 0\right), \\
\cdot & \cdot \cdot \cdot \cdot \cdot \cdot \cdot \\
f_{n} & =f\left(t_{1}, \ldots, t_{n-1}, t\right)
\end{aligned}
$$


ON $C R$-MAPPINGS BETWEEN ALGEBRAIC CAUCHY-RIEMANN MANIFOLDS 777

are algebraic in $t$ and holomorphic in other arguments. In order to prove Theorem 2 it suffices to show the algebraicity of these functions in all their arguments. We shall proceed by induction on $i$ (the number of the function $f_{i}$ in (5.4)). However, first we need some preliminaries.

Let $\alpha=\left(\alpha_{1}, \ldots, \alpha_{n}\right)$ be an integer multi-index and $|\alpha|=\alpha_{1}+\ldots+\alpha_{n}$. We denote by $f_{\alpha}(z)$ the derivative

$$
f_{\alpha}=\frac{\partial^{|\alpha|} f}{\partial z_{1}^{\alpha_{1}} \ldots \partial z_{n}^{\alpha_{n}}} .
$$

Lemma 5.1. Under the assumptions of Theorem 2 one can find a smaller subdomain $D^{\prime} \subset D$ such that all derivatives $f_{\alpha}(z)$ are algebraic in $t_{m}$ after restriction to each curve of any family.

Proof. The family of curves (2.6) is non-singular, therefore the transformation (2.6) from $z_{1}, \ldots, z_{n}$ to $t_{m}, c_{1}^{(m)}, \ldots, c_{n-1}^{(m)}$ and the inverse are implemented by algebraic functions. Hence in place of (5.5) we can consider the derivatives

$$
f_{\alpha}=\frac{\partial^{\alpha_{1}+\ldots+\alpha_{n-1}} f}{\left.\partial c_{1}^{(m)^{\alpha_{1}}} \ldots \partial c_{n-1}^{(m)}\right)^{\alpha_{n-1}}}
$$

and prove their algebraicity in $t_{m}$. Differentiation by $t_{m}$ and transformation to the original variables $z_{1}, \ldots, z_{n}$ do not destroy their algebraicity.

In order to prove the algebraicity of the derivatives (5.6) we shall use the algebraicity of the function $f\left(t_{m}, c_{1}^{(m)}, \ldots, c_{n-1}^{(m)}\right)$ in $t_{m}$ for fixed values of the other arguments. This means that we have an irreducible polynomial $P(f, t)$ in the ring $\mathbb{C}[f, t]$ of complex polynomials in the variables $f, t$ such that $f\left(t_{m}\right)$ satisfies the equation

$$
P\left(f\left(t_{m}\right), t_{m}\right) \equiv 0
$$

(see [VW, L]). Note that the coefficients of the polynomial (5.7) and even its degrees in $f$ and $t$ depend on the parameters $\left(c_{1}^{(m)}, \ldots, c_{n-1}^{(m)}\right)$. Let us define the following subsets in the range of values of these parameters:

$$
C_{q k}=\left\{\left(c_{1}^{(m)}, \ldots, c_{n-1}^{(m)}\right): \operatorname{deg}_{f} P=q, \operatorname{deg}_{t} P=k\right\} .
$$

The union of the countable number of $C_{q k}$ coincides with the whole range of values of the parameters $c_{1}^{(m)}, \ldots, c_{n-1}^{(m)}$. This allows us to use the following well-known Baire theorem.

Baire's Theorem. A complete metric space cannot be a countable union of nowhere dense subsets.

The proof can be found in [RS]. We apply this fact to the of range of parameters $c_{1}^{(m)}, \ldots, c_{n-1}^{(m)}$, and conclude that the closure of at least one of the sets $C_{q k}$ has nonempty interior. Choose a domain $D^{\prime}$ whose natural projection lies in the interior of such $C_{q k}$. Also, let us choose in $D^{\prime}$ a curve of the family (2.6) with parameters in $C_{q k}$. Without loss of generality one can assume that this curve corresponds to 
the parameters $c_{i}^{(m)}=0$ and the point $t_{m}=0$ on this curve is in $D^{\prime}$. For the polynomial (5.7) we have

$$
P(f, t)=\sum_{i=0}^{q} \sum_{j=0}^{k} a_{i j} f^{i} t^{j} .
$$

We normalize the polynomial (5.9) by setting some of its nonzero coefficients $a_{r s}$ to be equal to 1 . This polynomial vanishes after the substitution $f=f\left(t_{m}\right)$ and $t=t_{m}$. Let us consider the functions

$$
\varphi_{i j}=f(t)^{i} t^{j}, \text { where } i=0, \ldots, q \text { and } j=0, \ldots, k .
$$

They are algebraic in $t$ and depend holomorphically on the parameters $c_{i}^{(m)}$. If these parameters vanish, these functions (as functions of $t$ ) are linearly dependent. But elimination of the function $\varphi_{r s}$ with $a_{r s}=1$ makes the rest of the functions linearly independent. Otherwise we would have another nonzero polynomial $\tilde{P}(f, t)$ of the form (5.9) for which the equality (5.7) holds. Since $P$ is irreducible, we have $\tilde{P}(f, t)=u P(f, t)$, where $u \in \mathbb{C}[f, t] . \operatorname{But}_{\operatorname{deg}_{f}} \tilde{P} \leq \operatorname{deg}_{f} P$ and $\operatorname{deg}_{t} \tilde{P} \leq \operatorname{deg}_{t} P$, therefore $u \in \mathbb{C} \subset \mathbb{C}[f, t]$. Comparing the coefficients $\tilde{a}_{r s}=0$ and $a_{r s}=1$ we find that the equality $\tilde{P}(f, t)=u P(f, t)$ cannot be true for $u \neq 0$.

We denote by $X$ the set of all functions in (5.10), and by $X^{\prime}$ this set without $\varphi_{\text {rs }}$. Let us consider the Taylor expansions in $t$ of the functions (5.10). One can treat their coefficients as infinitely-dimensional vectors (columns) of the linear space $\mathbb{C}^{\infty}$. Such vectors corresponding to the functions from $X^{\prime}$ form the $\infty \times N$-matrix $A$, where $N$ denote the number of elements of $X^{\prime}$. The columns of $A$ are linearly independent if $c_{i}^{(m)}=0$. Therefore, there is an $N \times N$-submatrix $\tilde{A}$ of $A$ with non-zero determinant. This minor is holomorphic in $c_{i}^{(m)}$ and therefore does not vanish in a neighborhood of the origin. Hence, the columns of $A$ and the functions in $X^{\prime}$ are linearly independent for $c_{i}^{(m)}$ in a neighborhood of the origin.

Let us add the last column $B$ corresponding to the function $\varphi_{r s}$ to the matrix $A$, and consider the minors of order $(N+1)$ of the extended matrix $A \mid B$. They vanish for $c_{i}^{(m)}=0$ and for the parameters from the dense set $C_{q k}$. Therefore, they vanish identically. Thus, the functions in $X^{\prime}$ are linearly independent and the functions in $X$ are linearly dependent for every $c_{i}^{(m)}$ in a neighborhood of the origin. Thus, $\varphi_{r s}$ is a linear combination of the functions in $X^{\prime}$. Its coefficients up to the sign coincide with the coefficients of the polynomial (5.9). They are defined uniquely by the linear system with the extended matrix $(\tilde{A} \mid \tilde{B})$, where the $N$-th column $\tilde{B}$ is formed by the elements of $B$ lying in the rows defining $\tilde{A}$. Thus, the coefficients of the polynomial (5.9) are holomorphic in $c_{i}^{(m)}$, on a neighborhood of the origin. Now one can differentiate the equation (5.7) with respect to $c_{i}^{(m)}$, and we easily complete the proof.

Let us consider the functions (5.4). One can shrink $D$ to $D^{\prime} \subset D$ according to Lemma 5.1. Also, one can assume that the degrees of the polynomials (5.7) in $f$ and $t_{m}$ depend only on $m$ in $D^{\prime}$. Choose a point $z^{0}$ from (5.3) in the domain $D^{\prime}$. This determines the functions (5.4). For the function $f_{1}$ Lemma 5.1 gives the algebraicity in $t$ of the derivatives

$$
\left.\frac{\partial^{s} f}{\partial t_{2}^{s}}\right|_{(t, 0, \ldots, 0)}
$$


The derivatives (5.11) coincide with the derivatives of the function $f_{2}$ from (5.4) for $t=0$. In fact,

$$
\left.\frac{\partial^{s} f_{2}\left(t_{1}, t, 0, \ldots, 0\right)}{\partial t^{s}}\right|_{t=0}=\left.\frac{\partial^{s} f}{\partial t_{2}^{s}}\right|_{\left(t_{1}, 0, \ldots, 0\right)} .
$$

We need the following

Lemma 5.2. An algebraic function $f(t)$ is defined uniquely by its value and the values of a finite number of its derivatives at a regular point. If these values depend algebraically on a parameter $\tau$, then $f=f(t, \tau)$ is an algebraic function of both variables $t$ and $\tau$.

Assume the defining irreducible polynomial of the algebraic function $f(t)$ has the form (5.9). Repeating the above arguments, we again consider the functions (5.10) and their Taylor expansions at a regular point (one can assume it to be $t=0$ ). The coefficients of these expansions depend algebraically on $f$ and its derivatives at $t=0$. Considering as above a nonsingular submatrix $\tilde{A}$ and the corresponding linear system, we apply the Cramer rule and get the coefficients of the polynomial (5.9). In the second hypothesis of our claim they are algebraic in $\tau$. By the separate algebraicity principle we conclude the argument.

We apply Lemma 5.2 to the function $f_{2}\left(t_{1}, t, 0, \ldots, 0\right)$, taking into account its algebraicity in $t$ and the algebraicity of derivatives (5.12) in $t_{1}$. Therefore, the function $f_{2}\left(t_{1}, t, 0, \ldots, 0\right)$ is algebraic in both variables.

This is the first induction step.

Assume that the functions $f_{1}, \ldots, f_{m}$ in (5.4) are algebraic. It follows from Lemma 5.1 that the derivatives

$$
\left.\frac{\partial^{s} f}{\partial t_{m+1}^{s}}\right|_{\left(t_{1}, \ldots, t_{m}, 0, \ldots, 0\right)}=\left.\frac{\partial^{s} f_{m+1}\left(t_{1}, \ldots, t_{m+1}, 0, \ldots, 0\right)}{\partial t_{m+1}^{s}}\right|_{t_{m+1}=0}
$$

are algebraic as well. Lemma 5.2 and the algebraicity of the derivatives (5.13) in $t_{1}, \ldots, t_{m}$ give the induction step from $m$ to $m+1$. This completes the proof of Theorem 2 .

\section{REFERENCES}

[Al] H.Alexander, Holomorphic mappings from the ball and polydisk, Math. Ann. 209 (1974), 249-256. MR 50:5018

[BM] S.Bochner and W.T.Martin, Several complex variables, Princeton University Press, Princeton, 1948. MR 10:366a

[BP] A.Boggess and J.Polking, Holomorphic extension of CR functions, Duke Math. J. 49 (1982), 757-784. MR 84j:32018

[Ch] E.Chirka, Introduction to the geometry of CR manifolds, Uspehi Mat. Nauk 46 (1991), 81-164. MR 92m:32012

[Fo] F.Forstneric, Mappings of quadric Cauchy - Riemann manifolds, Math. Ann. 292 (1992), 163-180. MR 93e:32033

[L] S.Lang, Algebra, Addison-Wesley, Reading, MA, 1965.

[Pe] D.Pelles, Proper holomorphic self-maps of the unit ball, Math. Ann. 190 (1971), 298-305. MR 43:3501

[Pi] S.I.Pinchuk, Boundary uniqueness theorem for holomorphic functions of several complex variables, Mat. Zametki 15 (1974), 205-215. MR 50:2558 
[Po] H.Poincaré, Les fonctions analytiques de deux variables et la representation conforme, Rend. Circ. Mat. Palermo 23 (1907), 185-220.

[RS] M.Reed and B.Simon, Methods of modern mathematical physics. V 1. Functional analysis, Academic Press, New York and London, 1972. MR 58:12429a

[Ru] W.Rudin, Function Theory on the Unit Ball of $\mathbb{C}^{n}$, Springer, New York, 1980. MR 82i:32002

[Su1] A.Sukhov, On CR mappings of real quadric manifolds, Michigan Math. J. 41 (1994), 143-150. MR 94:08

[Su2] - On holomorphic mappings of domains of the type of "wedge", Mat. Zametki 52 (1992), 141-145. MR 94e:32047

[Su3] On the mapping problem for quadric Cauchy - Riemann manifolds, Indiana Univ. Math. J. 42 (1993), 27-36. MR 94e:32039

[Su4] On algebraicity of complex analytic sets, Math. USSR Sbornik 74 (1991), 419-426. MR 93e: 32014

[Ta] N.Tanaka, On the pseudo-conformal geometry of hypersurfaces of the space of $n$ complex variables, J. Math. Soc. Japan 14 (1962), 397-429. MR 26:3086

[TH] A.Tumanov and G.Henkin, Local characterization of holomorphic automorphisms of Siegel domains, Funct. Anal. and Appl. 17 (1983), 49-61.

[Tu] A.Tumanov, Finite dimensionality of the group of $C R$ automorphisms of standard $C R$ manifolds and proper holomorphic mappings of Siegel domains, Izv. Akad. Nauk USSR Ser. Mat. 52 (1988), 651-659. MR 89f:32061

[VW] B.L.Van der Waerden, Algebra 1, Springer Verlag, Berlin-Heidelberg-New York, 1966. MR 41:8186

[W1] S.Webster, On the mapping problem for algebraic real hypersurfaces, Invent. Math. 43 (1977), 53-68. MR 57:3431

[W2] - Holomorphic mappings of domains with generic corners, Proc. Amer. Math. Soc. 86 (1982), 236-240. MR 83k:32041

Department of Mathematics, Bashkir State University, Frunze street 32, 450074 Ufa, RusSia

E-mail address: yavdat@bgua.bashkiria.su

CMi, Universite de Provence, 39 Rue F. Joliot-Curie, 13453 Marseille Cedex 13, FRANCE 